\title{
Anaphylaxis after administration of amikacin containing sodium metabisulfite in a premature newborn
}

\author{
Pinar Kendigelen, $M D^{a}$; Mehmet Baktir, $M D^{b}$; Asena Sucu, $M D^{c}$ and Prof. Guner Kaya, $M D^{a}$
}

\begin{abstract}
Anaphylaxis is a serious systemic hypersensitivity reaction that is rapid in onset and can cause death. Premature newborns, whose immunological system is immature, are less likely to develop anaphylaxis. Administration of amikacin, containing sodium metabisulfite, to a 3-day-old premature newborn, induced a near fatal anaphylaxis. After suspicion of sepsis, the baby was started on amikacin. Clinical improvement was observed after initiation of treatment. On the third day of treatment with amikacin, the newborn suddenly developed tachypnea, tachycardia, angioedema and cyanosis. Anaphylaxis was diagnosed and treated. Latent reaction occurred after one hour of clinical improvement. The baby was intubated immediately. Anaphylaxis is a medical emergency; therefore the clinicians should have a rapid and careful assessment about this potentially fatal reaction. Even after successful treatment of anaphylaxis, the patient should be under observation for 72 hours because of the possibility of a biphasic reaction.

Key words:anaphylaxis; amikacin; sodium Metabisulfite; premature infants.
\end{abstract}

http:/ / dx.doi.org/10.5546/aap.2016.eng.e195

\section{INTRODUCTION}

Anaphylaxis is a medical emergency; therefore the clinicians should have a careful and rapid assessment about this potentially lethal reaction. Hypersensitivity reactions can occur in children, even under 6 months of age, whose immunological system is still immature. Amikacin is a semi-synthetic aminoglycoside antibiotic which has low incidence of anaphylaxis

a. Department of Anesthesiology and Intensive Care, Cerrahpasa Medical School, Istanbul University, Istanbul, Turkiye.

b. Clinic Of Anesthesiology And Reanimation, Afsin State Hospital, AfsinKahramanmaras,Turkiye.

c. Clinic Of Pediatrics, Afsin State Hospital, AfsinKahramanmaras, Turkiye.

E-mail address:

Pinar Kendigelen, M.D.: pinarken@gmail.com

Funding: None.

Conflict of interest: None.

Received: 10-22-2015

Accepted: 11-30-2015 induction. Preservatives, which are present in drugs, may cause anaphylaxis. We present a premature newborn who developed biphasic anaphylaxis following intravenous treatment with sodium metabisulfite-containing amikacin.

\section{CASE REPORT}

A male baby was born via spontaneous vaginal delivery at 33 gestational weeks. The mother was a 22 years-old primigravid. This preterm delivery occurred after premature membrane rupture. The birth weight was $1900 \mathrm{~g}$ and the Apgar score was 10 at 5 minutes after birth. He developed hypotonicity 3 hours later. Premature delivery, premature membrane rupture, and hypotonicity were interpreted as early-onset sepsis (EOS) signs. Blood samples were obtained for hemoculture. Intravenous ampicillin and amikacin were administered.

The EOS symptoms disappeared after two days of antibiotic treatment. At third day of antibiotic treatment (12 hours after the administration of ampicillin), during intravenous amikacin infusion, the newborn suddenly developed tachypnea, tachycardia and mild angioedema (Figure 1). Infusion with amikacin was discontinued when anaphylaxis was suspected. The baby was ventilated with a bag-valve mask supplying $100 \%$ oxygen. Respiratory and circulatory monitoring was conducted. An intramuscular adrenaline (1:1000) $0.01 \mathrm{mg} / \mathrm{kg}$ injection was provided. A $20 \mathrm{~mL} / \mathrm{kg}$ intravenous dose of $0.9 \% \mathrm{NaCl}$ was administered to treat hypovolemia. Clinical improvement was documented. Hemodynamic stability was established. The second line treatment performed consisted of intravenous diphenhydramine $1 \mathrm{mg} / \mathrm{kg}$, methylprednisolone $2 \mathrm{mg} / \mathrm{kg}$ and ranitidine $1 \mathrm{mg} /$ $\mathrm{kg}$. Serial arterial blood sampling was conducted for the diagnosis and treatment of metabolic acidosis. Hypoglycemia and hypothermia were avoided. There were not hypoxic events. The baby was monitored for spontaneous breathing. The above clinical condition and response to treatment was interpreted as anaphylaxis. One hour later, he developed acute hypotension, tachypnea, severe angioedema and cyanosis (Figure 2). After 
latent reaction was diagnosed, an intramuscular adrenaline (1:1000) $0.01 \mathrm{mg} / \mathrm{kg}$ dose was repeated. The newborn was intubated and $100 \%$ oxygen was delivered via an endotracheal tube. A second dose of $20 \mathrm{ml} / \mathrm{kg} 0.9 \% \mathrm{NaCl}$ was infused intravenously. Diphenhydramine $1 \mathrm{mg} / \mathrm{kg}$, methylprednisolone $2 \mathrm{mg} / \mathrm{kg}$ and nebulized salbutamol were also administered. After this treatment the signs and symptoms of latent reaction were completely resolved. Hemodynamic stability was again established. Since the initial hospital was a first level center, after intubation he was transferred to the Neonatal Intensive Care Unit (NICU) at a different location (Figure 3). He stayed on mechanical ventilation for one day and was extubated without complications. The baby did not have signs of sepsis and hemoculture results were negative, laboratory data was normal and antibiotics treatment were stopped. Hemodynamic and respiratory parameters were stable on the follow-ups. He was discharged from the NICU 3 days later. Long-term follow up of the patient did not reveal any other unexpected events.

FiguRe 1. First Reaction

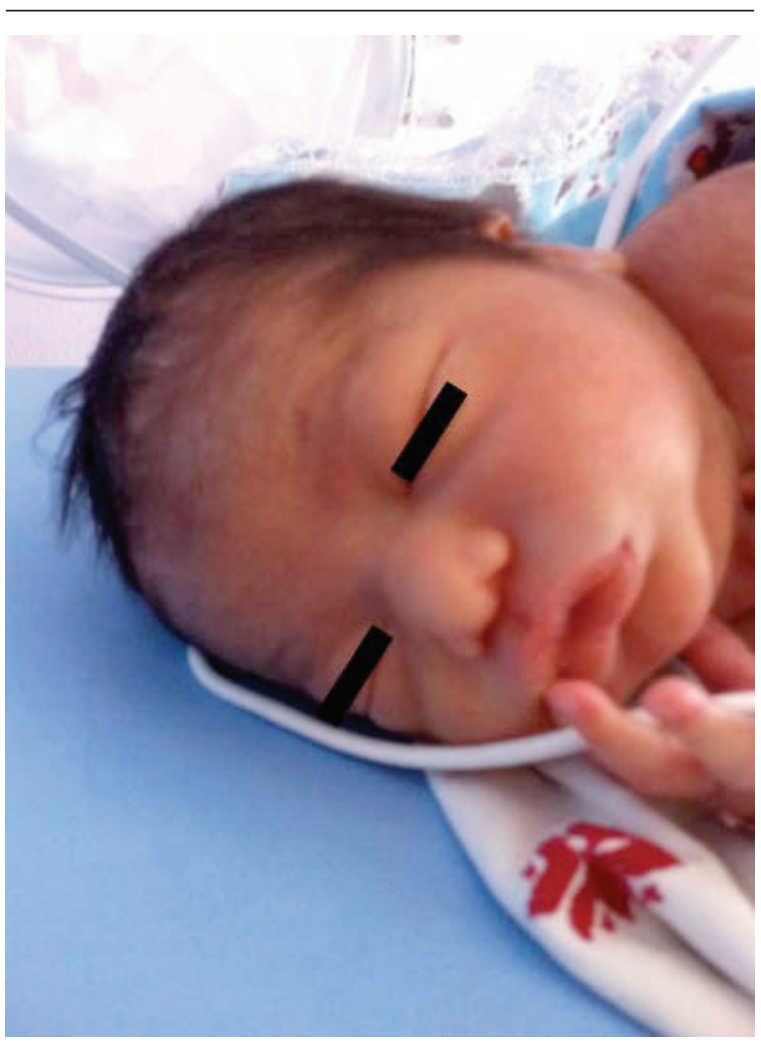

\section{DISCUSSION}

Anaphylaxis is an acute and severe type I hypersensitivity reaction characterized by rapid onset. The most frequent triggers of anaphylaxis in children include food, insect venom, drugs, preservatives and additives. ${ }^{1}$ Anaphylaxis clinical diagnosis is based on physical signs and findings, history of exposure to a triggering agent and onset of symptoms and signs over minutes or hours (i.e. flushing; generalized edema, prominent in tongue and lips; dyspnea; bronchospasm; reduced blood pressure; associated symptoms). ${ }^{2}$ Immune system gains sensitivity after exposure to an allergen. During the exposure to the same agent for the second time, the immunoglobulin E (IgE) binds the antigen and this complex stimulates to release mediators from basophils and mast cells. These mediators cause vasodilation, laryngeal edema, bronchoconstriction, platelet aggregation and increased capillary permeability. The most common systems affected in anaphylaxis include the cutaneous, respiratory, cardiovascular and gastrointestinal systems..$^{2-3}$ Clinical signs and findings for the diagnosis of anaphylaxis are

FIGURE 2. Latent reaction (flushing in the face area, edema which was prominent in tongue and lip)

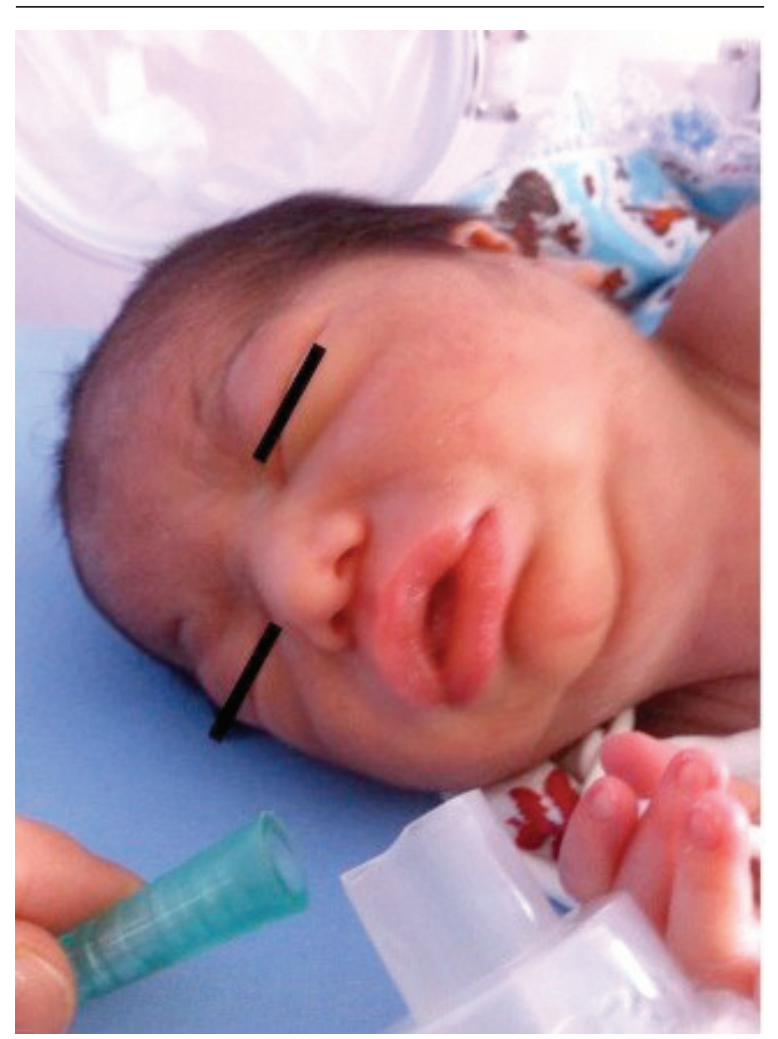


always superior to laboratory tests, such as plasma histamine and tryptase levels. ${ }^{4}$ Successful management of anaphylaxis is based on early diagnosis and treatment. This includes verifying airway obstruction and making sure that breathing and circulation is supported. Early elective intubation is suggested in presence of stridor, hoarseness, and edema in tongue and oropharynx. Since the edema may progress rapidly and intubation gets more difficult throughout time, intubation under sedation may be safer ${ }^{5,6}$ If there are concerning signs in regards to circulation and respiration, adrenaline is the first choice of treatment. Oxygen therapy, fluid restoration, antihistamines, beta-agonists and corticosteroids are also part of the supportive treatment. ${ }^{7}$ Patients should be followed up after treatment. The follow-up duration is based on the strength of allergic reaction, whether there is history of asthma and biphasic reaction or if there is continuing exposure to the antigen. Since the biphasic reaction may be observed in $20 \%$ of patients in a 72 hour period, it is recommended that patients should be followed up to at least 72 hours. Biphasic allergic reactions may not be understood well, but they are more frequently

FIGURE 3. After intubation

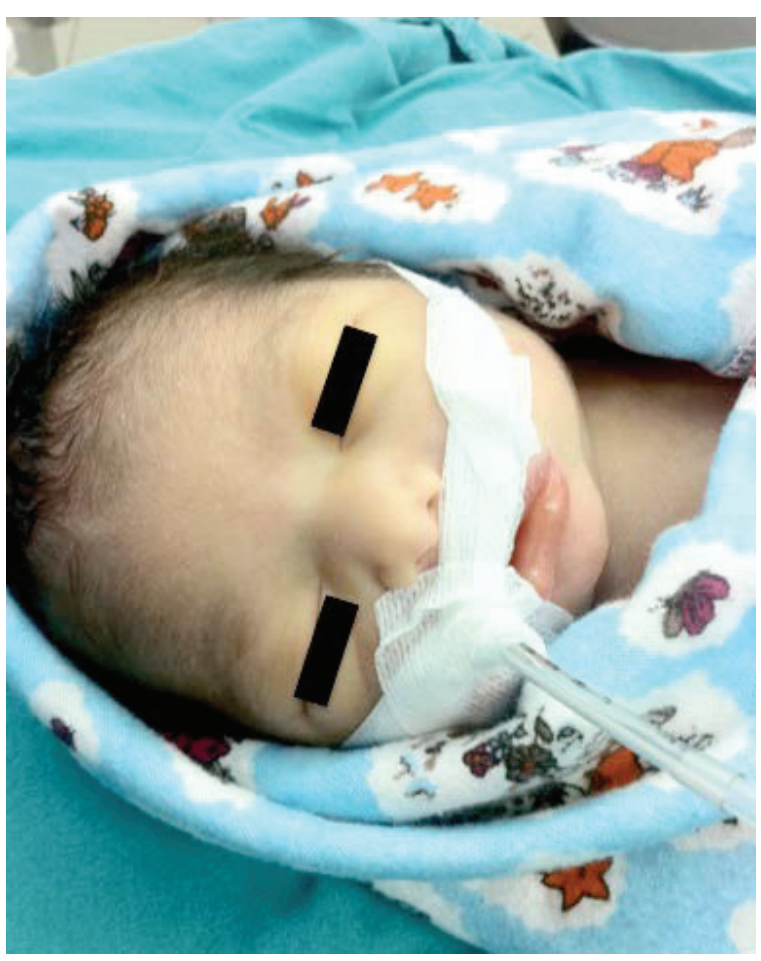

seen in patients who had their first reaction with severe symptoms. ${ }^{5}$

We observed the above mentioned clinical signs and symptoms of first reactions of anaphylaxis during intravenous infusion of sodium metabisulfite-containing amikacin in a preterm male infant. This newborn responded well to the medical treatment provided. Another suspected agent was ampicillin but, after a retrospective analysis of the patient's record, the type of injections, time, and drugs suggest that the only possible responsible agent was sodium metabisulfite-containing amikacin. We did not identify a specific allergic response to any other agents and drugs in the family members.

Amikacin is a semi-synthetic aminoglycoside antibiotic and amikacin-related anaphylaxis is very rare. To our knowledge, anaphylaxis after intravenous amikacin infusion was only reported once in an adult. The authors suspected that preservatives like methylparaben and propylparaben, which were present in the amikacin drug, may cause anaphylaxis. ${ }^{8}$ In our case, the amikacin preparation (Amijeksin, [TÜM-EKIP, Istanbul, Turkey]) included sodium metabisulfite, which is a common preservative used in many different types of foods and drugs. ${ }^{9}$ Anaphylactic reactions related to sulfite local anesthetics, gentamicin, and others medications have been previously reported. ${ }^{10}$

Hypersensitivity reactions can occur in children even under 6 months of age, whose immunological systems are still immature. ${ }^{11}$ Our case is one of the rare cases on newborn anaphylaxis. ${ }^{11-14}$ Because of their immature immune system, diagnosis of anaphylaxis in newborns is more challenging. In addition to that, some anaphylaxis signs like flushing and dysphonia can be seen in healthy crying babies. Differential diagnosis should be done in life threatening cases like foreign object aspiration, respiratory and gastrointestinal malformations and sudden baby death syndrome.${ }^{15}$ Hereditary angioedema(HE) has life threatening symptoms of upper airway obstruction which resembles anaphylaxis. However, HE is unresponsive to adrenaline, antihistamines or steroids. Furthermore, HE patients usually have family history that our patient does not have. Since our patient was premature, and had a low risk of developing hypersensitivity reaction (i.e. immature immune system), symptoms like tachycardia and tachypnea, are generally seen in septic shock observed during neonatal sepsis. 
However, anaphylaxis was strongly suspected, because of the sudden onset after treatment with amikacin, along with the clinical signs of tachypnea, hypotension, tachycardia, bradycardia and angioedema.

The diagnosis of anaphylaxis of a newborn is more difficult than in children because of their immature immune system. Anaphylaxis is a medical emergency; therefore clinicians should be able to perform a rapid and careful assessment in regards to this potentially fatal reaction in the newborn. This should include monitoring and maintenance of the airways, breathing and circulation. Since a latent reaction may be more serious than the first reaction, even after successful treatment of anaphylaxis, the patient should be observed 72 hours for possibility of a biphasic reaction.

\section{REFERENCE}

1. Ben-Shoshan M, Clarke AE. Anaphylaxis: past, present and future. Allergy. 2011;66(1):1-14.

2. Topal E, Bakirtas A, Yilmaz O, Ertoy Karagöl IH, et al. Severe anaphylaxis in children: a single-center experience. Pediatr Neonatol. 2014;55(4):320-2.

3. Sampson HA, Muñoz-Furlong A, Campbell RL, Adkinson $\mathrm{NF}$ Jr, et al. Second symposium on the definition and management of anaphylaxis: summary report-Second National Institute of Allergy and Infectious Disease/Food Allergy and Anaphylaxis Network Symposium. J Allergy
Clin Immunol 2006;117(2):391-7.

4. Simons FE. Anaphylaxis: Recent advances in assessment and treatment. J Allergy Clin Immunol. 2009;124(4):625-36.

5. Lane RD, Bolte RG. Pediatric anaphylaxis. Pediatr Emerg Care. 2007;23(1):49-56.

6. Liberman DB, Teach SJ. Management of anaphylaxis in children. Pediatr Emerg Care. 2008;24(12):861-6.

7. Samanta S, Samanta S, Jha A. Amikacin-triggered anaphylaxis: Should we go for skin test? J Pharmacol Pharmacother. 2014;5(1):53-4.

8. FuzakJK1, Trainor J. Comparison of the incidence, etiology, and management of anaphylaxis over time. Pediatr Emerg Care. 2013;29(2):131-5.

9. Bush RK, TaylorSL. Reactions to Food and Drug Additives. In: Adkinson NF Jr, Bochner B, Burks AW, Busse WW, et al. Middleton's Allergy Principles and Practice. 8thed.Philadelphia: Elsevier Publishing; 2014:1340-55.

10. Smolinske SC. Review of parenteral sulfite reactions. J Toxicol Clin Toxicol. 1992;30(4):597-606.

11. Carder KR. Hypersensitivity reactions in neonates and infants. Dermatol Ther 2005;18(2):160-75.

12. Bulbul A, Karadag A, Köklü E, Pamuk U, Sarici SU. Anaphylactic shock due to hepatitis B immunoglobulin in a newborn. J Matern Fetal Neonatal Med. 2010;23(10):1257-9.

13. Babu TA, Sharmila V. Cefotaxime-induced near-fatal anaphylaxis in a neonate: A case report and reiew of literature. Indian J Pharmacol. 2011;43(5):611-2.

14. Babakissa C, Lemire C, Beaulieu G, Ferretti E. Hypersensitivity reaction to parenteral nutrition in an intrauterine growth-restricted newborn: a case report. JPEN J Parenter Enteral Nutr. 2011;35(3):402-4.

15. Simons FE. Anaphylaxis in infants: can recognition and management be improved? J Allergy Clin Immunol. 2007;120(3):537-40. 